\title{
When Technology Disrupts Teaching and Learning: A Private Higher Education Students' Perceptions on the Challenges of Technology-based Teaching Tool
}

\author{
Reynold Padagas ${ }^{1, *}$, Ruth Gutierrez ${ }^{1}$, Lucero Trangia ${ }^{2}$ \\ ${ }^{1}$ College of Liberal Arts, Criminology and Education, Jose Rizal University, Philippines \\ ${ }^{2}$ Junior High School Division, Jose Rizal University, Philippines
}

Received August 17, 2019; Revised September 27, 2019; Accepted October 6, 2019

Copyright $\bigcirc 2019$ by authors, all rights reserved. Authors agree that this article remains permanently open access under the terms of the Creative Commons Attribution License 4.0 International License

\begin{abstract}
Higher Education Institutions (HEIs) have always been confronted by several conflicting views of whether or not online platforms are indeed efficient and effective methods of quality teaching and learning since information age renewed almost all aspects of human development. The pedagogy, curriculum, and assessment of student learning become the center of many discussions as to how online platforms recalibrate and accelerate quality education. Putting up students and teachers in the cloud accentuated debates, but this never stops HEIs to push through with the use of online platforms. To improve delivery, the online platforms must intensify co-creation among the students and teachers to maximize utilization despite of reverberating challenges. This exploratory research revealed several challenges encountered by 22 students in blended learning classes utilizing Canvas as a major platform. Course of actions are proposed to revitalize the utilization of Canvas despite of pedagogical, curricular, and learning assessment adversities. As posited, technology is a means to an end (Heidegger, 1954).
\end{abstract}

Keywords Canvas, Pedagogy, Curriculum, Assessment of Learning

\section{Introduction}

The continuous use of online platforms is changing educational principles on effective teaching designed to improve students' learning progress. Online platforms such as the Learning Management System (LMS) continuously affect the way education is enforced among students in class. According to Aldiab et al. (2019), there is a strong global trend toward utilizing LMS in academic institutions as a part of their educational management system to improve the teaching and learning experience in higher education system. Teachers in colleges and universities across the Philippines and the globe incorporate the LMS as a mode of instruction. Intensive training of university faculty members and students in the employment of the LMS in an optimal way increases its effectiveness in teaching and learning (Alshorman \& Bawaneh, 2018).

While many education experts believe that the use of LMS is a response to the call for creative and innovative education in the 21 st century and beyond, they are the ones who challenge this idea. Some studies are concerned about whether or not the LMS really innovates or it only digitizes the transmission of information in class.

Furthermore, the use of LMS poses the need to investigate the multifarious effects of how the goals and objectives of learning are set, how the quality of instruction is upheld and how student performance is assessed to ensure quality learning. It is the assumption of this research that the transition from previous LMS using traditionalist approaches to teaching and learning to new technologically advanced learning system causes significant implications to pedagogy, instruction, and assessment of learning.

There is a wide, disturbing gap between what it is and what it should be. While it is true that schools innovate their learning technologies to be adaptive to changing educational paradigms, schools must also provide reasonable baseline to deliver learning with unquestionable quality. The transition from the traditional brick classrooms into technology-based learning provides significant impact to the way schools realize their educational goals and objectives. While the vision is clear, schools need to take a look on the idea of digitalized education from the perspectives of all stakeholders to whether or not schools are integrating technology for the purpose of raising the bars of academic excellence or just digitalizing lessons with no difference from the traditional 
ones.

There is no such thing like best technology, only most appropriate technology. Adopting a teaching innovation in schools is also the same thing. There must be perfect fitting and complementary blend between purpose and means. In fact, challenges on pedagogy, curriculum and assessment of learning become overwhelming and might defeat the very purpose of the infrastructures presumably thought to be best. Further, schools also need to determine readiness of stakeholders to be able to maximize the use of it particularly for teaching and learning purposes.

Canvas, like any other LMS, is faced with a number of challenges despite its capacity to improve both teaching and learning as claimed by various educational experts. It is essential for HEIs to take a look on best practices of other institutions utilizing blended or online learning with LMS like Canvas to be able to impact quality of teaching and learning. Considering all these challenges that beset educational institutions, HEIs need to become more responsive enough to leverage the quality of education. Setting high standards is good but it is also dependent on the characteristics of stakeholders involved most especially the teachers and students while embracing the thrusts of the HEIs.

Constructivism, as a philosophy, posits that learners must be taught to construct knowledge. In a university, construction of knowledge is indeed demanded among students. If there is clarity of goals and objectives, handling classes in the online will create better opportunities to translate desired ends into tangible results not only measured through paper-and-pencil.

This research project explored on the challenges of Canvas as an alternative instructional modality to student learning in a private university. This study is relevant to a private university's thrust to become a market leader of innovative teaching and learning. The results may benefit the university to enhance its capacity to fulfill the vision and mission set through the new learning management system.

The findings challenge the academic institution for its policy improvements. Through these policy improvements, the delivery of instruction to the students using Canvas will improve especially now that outcomes-based education (OBE) has been adapted by Higher Education Institutions (HEIs) towards quality student learning.

\subsection{Background of the Study}

The locale of this study is a private university in the Philippines. This University newly adopted Canvas as a software application to deliver and manage instructional content in various courses. Through Canvas, the teachers learn to organize their lessons through modules, create engaging and meaningful activities and facilitate several forms of learning assessments. Things, however, were not easy during the adoption of Canvas as the institutional online platform. The transition from a traditional classroom set-up to a technology-driven learning environment created some challenges in teaching and learning processes. Students, in particular, continuously cope with these challenges despite the disruptions that Canvas generated.

With the University's thrust to become a market leader of innovative teaching and learning, technological improvements such as Moodle and Canvas have been employed as online learning platforms. Canvas was introduced in the university in 2016 as the latest learning management system that is expected to provide meaningful learning experiences of university students. The University acquired Canvas to fill in the flaws of the previously used learning management system. This led the researchers to study about Canvas particularly students' challenges in using it as an online learning tool. At first, there were some hesitations in pursuing this study since Canvas was just newly adopted in the University. However, the researchers realized that if problems will be recognized early, university's investment will not be wasted hence the improvements in the way teachers and students utilize Canvas can impact quality learning.

The researchers had the opportunity to handle some of the courses using blended learning as a mode of instructional delivery. Based on the researcher's observations and the reports given to them by their students enrolled in other blended learning courses, it can be noted that complacency has become the practice of some students enrolled in Canvas due to some teachers' leniency, resistance or even ignorance in using the online platform. The quality of instruction, assessment of learning and curriculum is challenged.

The dramatic effect of information technology on society is undeniable. In education, it is evident in the use of terms like active learning, blended learning, electronic learning and mobile learning (ubiquitous learning) (Conradie, 2013).

Karoudis et al. (2016) cited that the past twelve years have seen ubiquitous learning (u-learning) emerging as a newlearning paradigm based on ubiquitous technology. By integrating a high level of mobility into the learning environment, u- learning enables learning not only through formal but also through informal and social learning modalities (Karoudis et al., 2016). Patel and Patel (2017) posited that both e-learning and in-person pedagogy has become more famous in higher education in recent years that is termed as blended learning.

LMS significantly affected the way teaching and learning are delivered. Stoica and Støckert (2018) conducted a study on new learning scenarios for multi-campus large classes in Mathematics. The main aim of the designed learning scenario is to bridge the geographical gaps by applying a novel blend of pedagogy and technology in order to create and support a learning space, which enables students' active learning and promotes efficient cross campus collaboration and 
communication.

Not only in General Education subjects are LMS applied. In fact, in the field of medicine and allied medical courses such as pharmacy, LMS has been proven effective and efficient in the overall learning experiences of students. As such, an online approach framed by educational theory can be an effective way to provide pedagogy instruction within a teaching program (Garrison et al., 2015). Further, Garrison et al. (2015) highlighted that a learning management system was used to assess the achievement of learning objectives and participation prior in progressing to the teaching experiences component of the teaching program.

Students have a positive outlook about LMS that could provide them the knowledge that they will need without any hassle in learning. Using their gadget, like cellphone, laptop, and computer desktop, they are confident that they could learn on how to use LMS. As student aged, they are more optimistic in using the LMS, likewise the higher the educational attainment of the student, the higher they perceived LMS usefulness and easiness in use. Using LMS as pedagogy is not a problem for them as long as they are familiar in the interface of the LMS that will be using. Constant using of LMS for familiarity can, as well, ease the use of LMS.

The LMS is very convenient for both the students and instructor in the online environment to organize communication, interaction, thread of discussions, sharing of files, forums, online monitoring of success and performance, and several statistical analyses. Through LMS, the role of the instructor is to facilitate and manage the learning of students by giving different kind of task like video conferencing, forums for discussion, taking examination and planning to deliver the content of the lesson. As a web based learning tool, the LMS should facilitate an accessible learning content for the student (Adzharuddin \& Ling, 2013).LMS provides promising opportunities for both students and professors. It serves as the bridge for students and professor in the teaching and learning process providing quality of education. LMS provides students a chance to learn information at their own pace provides forums and interaction with their classmates about the topic being discussed, they could get ideas gathered from different sources that could open for new knowledge that will be facilitated by their professor. LMS offers professors to easily track students' performance in every step of their way, check the achievement of students and have online results of examinations. LMS saves time of professors without sacrificing the quality of instruction. There are a lot of remunerations from LMS which includes self-paced learning for students, content can be repeatedly read until the learner absorbs the knowledge, e-learning is culture independent, flexibility of learning based on the timing and completion of syllabus, and individual problem solving is possible (Kulshrestha, 2013).
The goal of this study was only to explore on the challenges encountered by students with the use of Canvas as a learning tool in a private University. It is through their lens that recommendations are drawn since they are regarded as primary users of the said LMS. The researchers solely based their conclusions on the perceptions provided by the students during the conduct of this study.

\section{Materials and Methods}

\subsection{Research Design}

This study utilized descriptive qualitative exploratory research design to determine the challenges of Canvas in pedagogy, curriculum and assessment of learning among students in a Private University in the Philippines. The study was conducted in six months.

\subsection{Research Participants}

The participants were purposively selected from different colleges enrolled in courses using Canvas. Series of focus group discussion (FGD) were conducted depending on the availability of the participants. There were five participants from various courses (i.e. Natural Science, Computer Course, and Engineering Course) for the FGDs 1, 3, and 4. On the other hand, FGD 2 was composed of seven students (i.e. Freshmen Orientation Seminar).

\subsection{Research Instrument}

An FGD protocol was developed to gather data. The research director, their mentor, and some university professors validated the FGD protocol. The improved FGD protocol was pilot tested among students. Revisions were made to the protocol and eventually used in the data collection.

\subsection{Data Gathering Procedure}

The first FGD was conducted in the university library learning commons, while the rest of the FGDs were done in selected classrooms. The participants were properly briefed about on how to conduct the FGD and assured anonymity of their personal information. Some of the FGDs were quite challenging that required the researchers to translate questions in language that the participants were capable of comprehending.

\subsection{Treatment of Data}

Content analysis was made on the different transcripts of the actual FGD. Several themes were identified that encapsulate the findings of this study. The researchers 
highlighted the transcripts with rigor and integrity so that biases can be prevented in the analysis of the data. The rigor of this research was ensured by scrupulously adhering to a specific philosophical perspective, and thoroughness in collecting data. Meanwhile, the transferability of the findings is warranted to other educational contexts or settings.

\subsection{Ethical Considerations}

Informed consent was given to each of the participant. The participants ensured the confidentiality and anonymity. The record and data transcriptions collected will only be stored for about five years.

\section{Results and Discussions}

In the advent of advanced technology, the teaching and learning dynamics continuously change and evolve. The evolution from the classical ways of teaching to newest trends of handling target classes poses significant implications not only in terms of pedagogy, but in instruction and assessment of learning as a whole. Pedagogical approaches are endlessly challenged especially that both teachers and learners are put on the spot to a new way of teaching and learning subject content. The quality of instruction is fried with lots of questions seemingly related to "digitalized" education - of whether or not education is really enhanced or everything is just digitalized with no reasonable baseline. Further, the assessment of learning is confronted with several issues that deface the very purpose of integrating technology or there is innovation in teaching on a particular subject. The rudiments of learning assessment are facing tumultuous challenges hence, the transition in general, rapidly affects all stakeholders especially teachers and students if the quality of education is fully attained or not. It takes optimism to develop and to enhance a modality that institutions think best to deliver education with unquestionable quality.

\subsection{Student Perceptions on the Characteristics of Canvas}

1. Accessibility. Canvas has been characterized by the study participants in various descriptions. Canvas, according to the participants, embarks "anytime, anywhere learning." This speaks about accessibility. It is amplified in the use of Canvas in learning various course contents.

As such, one participant cited:

"Canvas is really a great help to students because we could access our modules and lessons through the internet without any hassle..." (John)

On the other hand, the accessibility lies much on the internet connectivity. Not all students have internet connection at home. The same participant mentioned:

"...Canvas still has more to improve since not all student are like, ahhhh, connected to the internet." (John)

2. Convenient and time-saving. Attending classes nowadays is challenging especially that traffic jam takes a lot of travel time among students. The endless concern regarding heavy traffic in Metro Manila ruins time. Canvas becomes an effective alternative instructional delivery to some of the participants. Its convenience is significantly favor students with dilemmas in commuting just to attend face-to-face classes on time.

3. Easy-to-use. Students are digital natives. They take benefit of what technology can offer. With regard to Canvas as a mode of learning, students find it easy to use it for their advantage.

4. Helpful. The diversity of students in the University in terms of their socio-demographics and classifications matter. Some students are working to support their financial needs to attend school. Canvas is cited to be very helpful to them since missed discussions during the face-to-face learning can be remedied through the online materials. For a mother who still continues to fulfill her dream of getting a degree, Canvas is of great help.

"For me Canvas is a great help. Because like me I'm already a mother of two. So, I am able to open it anytime." (Nikki)

5. Innovation. Canvas has been described by the participants as an innovation. The University envisions itself to become a market leader in technology integration and innovation to effectively transfer knowledge. Canvas is predetermined as a teaching innovation that could harness transfer of learning to target classes.

6. Flexibility. The participants characterized Canvas as flexible and manageable since subject content tends to be modular.

7. No book requirement. Contrary to what some of the participants emphasized, Canvas still requires use of textbooks or references necessary to increases academic understanding of concepts. However, some participants perceived that there are no more book requirements in the use of it.

\subsection{Perceptions of Students about the Characteristics of Students Using Canvas}

1. Responsible. Most of the participants cited that they are becoming more responsible in terms of their learning through Canvas. They diligently check online lessons on a regular basis.

2. Independent. Students of Higher Education Institutions (HEIs) are expected to embrace 
independent learning. The Theory of Andragogy by Malcolm Knowles (1984) cited that adult learners are self-regulated and independent.

Student personality is also imperative to note. Self-regulated learning processes works best to some. Taking the initiative to compensate for their poor understand of course content is necessary to be able to learn better. The sense of responsibility is fostered but this only applies to some students.

One of the participants mentioned:

"I do not depend in Canvas, which is my personality. It's like, as a student, you should be responsible, if there are no answers from Canvas, you should find a way to answer the question." (Wale)

3. Can manage. The ability to manage utilization of Canvas is one important characteristic of the students. This, however, requires guidance and monitoring by teachers handling subjects using Canvas.

There are instances that students really have no choice but to understand lessons on their own. Independent learning is fostered. Managing one's self to learn is an integral function of an active learner.

One of the participants cited that:

"I couldn't really understand what's in the, what do you call this? In the lesson or in the process that is the time I'm going to search it via Youtube on how to do it? But just in any case that, just in any case, that, for example I'm really having a hard time to do it that is the time that I'm going to ask someone but for me, for me the best thing to do is to do it on my own." (Nikki)

Conversely, to some students, they claimed that they can really manage to pass their quizzes for as long as they read their online lessons. As such, a participant said:

"It is already your fault if you get a low score in Canvas." (Wale)

4. Procrastination. While Canvas assumes to leverage quality of learning. Some participants tend to procrastinate in using Canvas. Since quizzes, examinations and forums are set in a given period of time, students have the possibility to delay answering or submitting required tasks.

5. Prone to academic dishonesty. Canvas has number of pros. On the other hand, it has been brought out by some participants that academic dishonesty in terms of cheating and plagiarism are common issues confronting quality of learning outcomes. This suggests that the administration must find interventions to eliminate such dilemma.

6. Ambivalence. There were mixed feelings in terms of acceptance of Canvas as a learning modality. This ambivalence unleashes several issues in terms of Canvas it is an effective measure or not to level up quality learning. This poses concern to teachers as well. The acceptance of Canvas as an innovation in teaching and learning can be misperceived by learners when teachers contradict its use the way they wanted to deliver their instruction.

Some students prefer face-to-face rather than using Canvas. This happens because some professors also prefer such. They really have concerns on reading long slides present in Canvas lessons. The students also prefer to answer quizzes online rather than learning online because face-to-face enhances their cognitive skills. Some participants also cited that student learning styles are auditory rather than visual.

However, some students prefer the online learning through Canvas. It is considered favorable to them. When the professor is reliant in using Canvas, they take advantage of Canvas.

Regarding online engagement in Canvas, the participants cited that it is facilitated through forums, quizzes and assignments or requirements as cited by the participants.

As regard assessment of their levels of knowledge and skills, this is done through seat works during the face-to-face learning, quizzes, examinations, forum discussions, and student feedback.

The participants emphasized that to be able for students to get better grades, quizzes and examinations must be answered on time. Scores in all these aspects must also be high enough. There is a need to complete all forum discussions. There is a great need to follow-through instructions and steps given to accomplish assigned tasks. Doing all tasks ahead of time is also necessary. Opening or checking Canvas regularly to review lessons can not only improve understanding of complex lessons but also it is reflected to improved grades.

\subsection{Perceived Specific Challenges of Canvas}

\subsubsection{Forum-related Challenges}

Some forums are not easy to understand. Students, also, cannot follow through instructions. At times, tasks are done but not totally understood. Students also tend to cut-and-paste materials from unreliable resources such as the Wikipedia. Students just copy, reread, edit, and submit answers to tasks and forums.

\subsubsection{Student-related Challenges}

Some students are becoming forgetful of tasks needed to be accomplished in Canvas. Exercises are forgotten and worst is, they do not read their lessons. Some participants cited that they read without comprehension. They cannot withstand long readings. So, they depend so much on face-to-face learning since the professor does not use Canvas or just reiterate what has been embedded in Canvas.

The participants highlighted that some tasks in Canvas require too much of their time, which are becoming hassle and stressful. There are also topics embedded that are too 
confusing that is only necessitating teacher intervention.

\subsubsection{Professor-related Challenges}

Teacher presence is an important contributing factor to facilitate quality learning outcome in the online class. The participants revealed that steps and directions given by professors are not clear since they were not the ones who prepared the lessons in Canvas. Some participants cited that students are asking about the tasks, however, some professors do not even know the tasks in Canvas. Some professors do not also teach during the face-to-face classes.

Regarding the face-to-face learning, some participants said that students are far more attending to their classes than their professors. Some professors also fail to explain important concepts. They also do not give passwords of quizzes causing the students to delay course requirement completions. Some professors, according to the participants are not even aware of Canvas as a whole.

On a side note, it is interesting that the participants highlighted the importance of a professor who also maximizes the use of Canvas to impact better learning.

\subsubsection{Challenges on Instructional Materials}

Learning materials, on the other hand, seem to be a dilemma in Canvas. Most of the teachers handling some courses using Canvas were not the ones who prepared course content. This poses significant challenges on the part of the teachers translated into student difficulty to appreciate and learn their lessons.

In one of the participants' responses, it was cited that:

"It's a good thing if your professor uploaded and then discuss the file that he uploaded in the Canvas or something and control using the world wide web like Google concept in knowing the certain topic." (Ally)

There is a dire need to retool teachers to improve their ways of handling classes when using Canvas. Faculty training programs should be sustainable enough to be able to cater those who are in great need in advancing themselves to maximize Canvas in teaching their target classes. In this way, incomprehensibility and resistance will be resolved. Not all teachers are into technology-based learning, yet there is a call to reinvent the way they teach in the traditional classrooms. If teachers are not retooled in the use of technology, meaningful student learning will never be attained.

Conversely, there seems to be a dilemma in terms of course content embedded in Canvas. The level of interest or motivation of students becomes a subject of concern since there is redundancy of course content to contents already taken by the students from their basic education.

"Sometimes I use my stock knowledge. I already studied that when I was in high school." (Wale)

One participant, however, contradicted such proposition and was quoted saying:

"But normally Sir, based from our lessons in Canvas, only searching using Google can answer the question, because we don't have prior knowledge prior to the topics." (Ongcoco)

\subsubsection{Quiz-related Challenges}

As regards quizzes and examinations, some of these are missing at times. There is also a mixed up of multiple choice examinations and quizzes. The quizzes and examinations, sometimes, according to the students are not aligned to their online lessons and concepts.

Some students consider examinations and quizzes are quite easy and do not measure exactly what has to be measured.

"Examinations that they provide are too easy." (Manolito)

Quizzes and examinations must be constructed scientifically. In the advent of OBE, setting high expectations in teaching and learning is necessary. Some of the participants mentioned that teachers give sets of questions that do not require comprehensive analysis and interpretation. It appears that students are not being challenged in terms of their learning assessment since questions posted for specific tests are too easy and poorly constructed.

One participant cited:

"It's like being made up... So basic answers are also basic... It's like I wanted to answer it or sometimes I'm lazy answering it." (Eunice)

Confusion is another concern. When students are assessed via online quizzes and examinations, they experience some confusion in terms of the questions given to them. Some of the given choices in certain items are not correct as perceived by some participants. In this sense, there seems to be a poor construction of test items that defeat the purpose of learning assessment.

"Quizzes for example, it is easy but there are instances that the question but the answer... the given answers were wrong... I do know the answer but it is not in the choices, so I do not know what to choose." (Jill)

When students become critical of the learning assessment, learning indeed is taking place and this too, would affect their trust towards the system or worst is, towards the teachers. Adequate preparation on learning assessment is required to teachers. Test items should be constructed in such a way that they really are meant for their desired purpose without creating confusion among the students who are taking them.

\subsubsection{Lesson-related Challenges}

The participants revealed that lessons in the online are not tackled very well. There are a lot of chaotic lessons. Some are boring in terms of the way they are being presented. They are quite long and not understandable.

As such, one participant said:

"It's quite hard to learn a lesson in Canvas, like if there is something that you could not understand, you can't ask someone." (John) 
Searching the internet is one coping mechanism that students use to understand some difficult lessons. Filtering information is another concern. So, students have the dire need of personal guidance.

"But, you could search in the internet, but asking someone or like asking someone who knows better about the topic is quite better than searching in the internet." (John)

This only suggests that student assistance is necessary to realize learning objectives. When a student learning in Canvas does not fully understand concepts embedded, a student naturally looks forward for the immediate responses to cater to his or her learning needs. This ensures learning niche.

Some participants are aware that not everything is provided in Canvas. When students are challenged in their learning process, skipping lessons is common. Students tend to skip lessons whenever they do not understand what they have learned. Similar participant was quoted saying:

"Canvas is like not all the lessons are there like there are times that I skip lessons because there are times that I do not understand the lessons like that. And usually, I just read it through but I don't fully understand what the meaning of the lessons are." (John)

Lessons constantly need updating. It also requires immediate posting to prevent untoward consequences such as cramming on the part of student. In this way, student learning is far more achieved. Late posting should be prevented.

"In a special subject, our professor will post an additional lesson and there is a file that we need to pass a day after the professor posted it, the tendency for us is to cram. We need to download the file and answer the questions... it is like even the file is uploaded and update, it still lacks time to pass it." (Randy)

Informing students that new materials are posted is also imperative. Conscious consideration of time allotment to comply with course requirements in Canvas must also be made for the effectiveness of quality learning.

One participant further capitalized:

"Lessons are uploaded late and it's difficult to upload the answered file... the task given since late or lack of time to post." (Christian)

\subsubsection{Student Assistance-related Problems}

Some participants cited that there is no one to ask about the questions in Canvas. This result is quite confusing since the researchers observed that Student Assistance is provided to ensure that users of the Canvas are guided. The lack of awareness on their part necessitates orientation or reorientation.

\subsubsection{Student Collaboration Challenges}

Students collaborate through hands-on activities, group activities, when topics are not familiar, and when asking classmates when one do not know what to do. Via group chat, what were encountered during quizzes and topics are discussed.

Regarding student collaboration, several issues have been raised by the participants. Some students tend to copy the works of their classmates. Issues on plagiarism and cheating emerge in this situation. Some students are also complacent to participate in group chats and discussions. With regards to groupings, some teachers do not rearrange group members since most activities are individual-based. This is a call for more student collaboration to foster better learning.

It is also interesting to note that student collaboration to some is not attained simply because they do not belong to a block section. Reaching out to their classmates poses some concern.

"I' $m$ an irregular student, and my classmates are all in a block section so I always work for myself." (Rowell)

Sense of belonging seems to be of concern as well. There must be some ways on how teachers improve their mechanisms through Canvas to facilitate student collaboration.

However, one participant blatantly expressed that:

"For me, there is no such thing as collaboration in Canvas, because you answer your exam on your own, you learn your lesson on your own, and the time that only collaborate with my classmates is to update. When is the deadline of our quiz? When is the deadline of our activity? And usually they ask what the answer on the quiz here is? What is the answer on the activity here, so the collaboration there is like nonsense because you actually using Canvas as an individual, so there are not, there are no group works in Canvas just you, yourself." (John)

This is also supported by another participant when it was cited that:

"We don't have, ahhhmmm, activity that needs collaboration in Canvas." (Dale)

Student collaboration needs to be addressed to bring out its desired ends particularly in student learning. It has to be maximized to effect learning among students.

\subsubsection{Student Behavioral Concerns}

These include inability to meet all requirements for a specific topic, preference on hands-on activity than reading online lessons, preference on face-to-face than online learning, and preference on looking/searching the net than reading online materials.

There is also an issue of complacency since learning materials are embedded in Canvas. As regard to quizzes, since there is specific time frame to take them, students tend to be unworried, hence, they procrastinate.

One participant cited that:

"Actually, I don't plan. I just visit the canvas. Aaahmmm...periodically to check if there's a quiz and I just answer the quiz if the deadline is near." (Ongcoco)

\subsubsection{Challenges on Collaboration with Professors}

Teacher-and-student collaboration is fostered when 
students try to reach their professors for consultations. With regards to quizzes and forum discussions deadline, students seek considerations for teachers to extend the time of submission of requirements. The participants cited that some professors reply to concerns once they messaged.

However, several challenges have been identified regarding this area and these include the following: a.) some professors are not replying right away to address student concerns; $b$.) some professors do not use Canvas very often; c.) some professors are not familiar of Canvas; d.) students are aware of Canvas but some professors do not utilize and maximize its use; and e.) most professors prefer reporting as a method of class discussions and no longer use Canvas.

Teacher presence must be ensured. Learning becomes more meaningful in the presence of a teacher who guides students as they learn concepts.

One participant emphasized:

"There are some topics that has been uploaded on Canvas that I do not understand, then it could be better if we can reach out your professor who uploaded it." (Raymond)

\subsubsection{Technical Challenges}

Challenges in terms of technical aspect are common when using online methods in delivering teaching. However, immediate interventions must be instituted to address them because technical challenges can hamper student progress in learning.

The participants cited some of them and these include problems related to inability of desktops, duplicated slides, mixed up presentations, file conversions since Canvas utilizes free software for some embedded materials, password and accessibility to online lessons.

Despite the many challenges rooted in the use of Canvas, students cope to meet requirements. Some of them reread chapter lessons during their free time. Others keep on updating themselves by checking online lessons and materials whenever necessary. In fact, some students should check online lessons either daily or weekly. Some students beat their deadlines for tasks and requirements. They also answer quizzes before they expire. Students are also creative enough to make their lesson outline for them to be guided in studying their subject contents. To understand some other course content, students do not only rely on lessons embedded in Canvas. Some would search the internet using Google and other online resources yet failed to mention use of ProQuest, and other online resources offered by the University library. Constant checking of word synonyms via the internet also help them to understand difficult words used in their lessons.

One participant cited:

"I always use Google Chrome, for example there is a question or there is a sentence that I could not understand, so what I am going to do is, sometimes I am opening or I am frequently opening Meriam Webster or other search engines so that I could understand.” (Nikki)

When teachers are not around during their face-to-face learning, students do self-study. Adult learners are self-directed learners. This is according to Knowles.

According to the participants, teachers also help in coping with identified challenges while using Canvas in their blended learning courses. Some teachers compensate for their absences by adding some lessons in online. There are also extensions on lesson discussions.

\subsection{Students' Perceived Recommendations to Improve use of Canvas}

There must be active engagement of learners to improve their learning capacities using Canvas. How students learn best must be given importance to help them become more active in their online engagement.

The participants suggested the following to improve the delivery of learning through Canvas.

1. The University should enhance internet connectivity to resolve system glitch.

2. There must be continuous updating of software used in Canvas.

3. Teacher presence must be intensified.

4. Continuous system maintenance must be ensured.

5. The professor needs to adjust the time when taking quizzes so that students can comply.

6. The professor needs to compensate for the missed quiz through performance task.

7. Ensure that online lessons, quizzes and examinations are aligned.

8. Students and teachers must regularly check Canvas.

9. The professor should teach the lesson first during the face-to-face before taking quiz online so that students can get better scores and grades.

10. Teacher as proctor is needed during examinations.

\subsection{Implications to Teaching and Learning}

In its essence, Canvas as a technology-based learning tool has a good purpose and that is to accelerate the quality of student's learning. When learning becomes meaningful to students through it, academic performance geometrically increases.

There is interlock among teaching pedagogy, curriculum and assessment of learning. The pedagogy comprises instruction in one way or another, is affected by Canvas. In terms of instructional materials or lesson materials, Canvas must be thoroughly reviewed for improvement. The teachers play very crucial roles in the preparation of instructional materials. This, at some point, will make them more responsible and liable to quality student learning. Instructional materials must be well-designed to maximize the usefulness of Canvas. Teachers, in general, must be retooled to reinvent themselves due to demands of the $21^{\text {st }}$ century classrooms. 
Meanwhile, the curriculum for various courses using Canvas and those that intend to use Canvas as an instructional delivery is being challenged. The applicability in using Canvas in a particular course requires tedious reviews to make learning delivery better. It is imperative to have curricular reviews to make sure that Canvas really fits for its desired purpose especially now that OBE has been adopted.

Further, the assessment of learning through summative tests and formative tests must not be taken for granted. The development of good test items is needed to be able to measure what should be measured in very scientific ways. Both content and the technical aspect of the preparation of test items require expertise from teachers who are so called masters of content.

The challenges continuously flow along these components that directly and indirectly affect student learning. These challenges are ubiquitous along the interlocking components affected by Canvas but these do not paralyze the very purpose of quality teaching and learning. Canvas should be taken as an innovation that can possibly enhance capacities of all stakeholders particularly the students who are the raison d'etre why schools are built, why teachers are hired, why curriculum has been created, why instructional materials are made, and why pedagogies are needed for some flexibility.

The educational paradigm keeps on shifting from common to uncommon means of teaching and learning. The end cannot always justify the means but the means can always justify the end. This, too, is true to the use of Canvas. Given this fact, a keen eye and a high degree of openness are necessary to address some blind spots. After all, Canvas like any other online platforms can never replace a good teacher but it is complementary to teacher's instructional capacity.

\section{Conclusions and Recommendations}

There is no such thing like best technology, only most appropriate technology. However, schools need to look on the idea of digitalized education from the perspectives of the stakeholders. Schools need to determine readiness of stakeholders to be able to maximize the use of it particularly for teaching and learning purposes. Quality teaching and learning is a co-responsibility of stakeholders. Having said all these, there must be a perfect fitting and complementary blending of purposes and means. As such, in his work titled, The Question Concerning Technology, Heidegger (1954) emphasized that technology is a means to an end.

Based on the students' perceptions, the researchers identified that the lack of readiness of the faculty members in using Canvas as mode of teaching both in pedagogical content knowledge, and in the use of technology is concluded to have caused the identified challenges in utilizing Canvas as a tool in teaching. Because of the lack of readiness of the teachers in using Canvas for teaching, many student problems on learning surfaced. Issues on pedagogy, curriculum, and assessment of student learning were raised necessitating immediate interventions.

It is recommended, therefore, that the following measures are to be done to improve delivery of teaching and learning through Canvas as a major online platform. Formalize teacher-training program to ensure effective utilization of Canvas as a tool of learning. A formalized mentor-mentee program must be done in which an experienced teacher should lead in the retooling of teacher competencies using Canvas. Teachers must be retooled to come up with more updated and relevant modules and course packs for Canvas lessons. Realignment of lesson objectives, content, and methods of assessment must be done. Teacher presence must be felt in the online learning environment.

A complementary blend between face-to-face and Canvas learning must be reinforced. Student and teacher collaboration must be intensified. There must be an improved internet connectivity by increasing bandwidth. Student feedback mechanisms regarding Canvas must be enhanced to keep improvements towards quality of learning. Further, research studies can be conducted along the areas explored in this study by using teachers as primary research participants.

\section{Acknowledgements}

The researchers would like to extend their gratitude to Jose Rizal University headed by Dr. Vicente K. Fabella, President, Dr. Barbara Wong-Fernandez, Former Research Director, and Dr. Auxencia Limjap, G-Res Mentor, for supporting them to conduct the study. Likewise, immense appreciation is extended to the student-participants.

\section{REFERENCES}

[1] Adzharuddin, N.A., \& Ling, L.H. (2013). Learning Management System (LMS) among university students: Does it work? IJEEEE 2013 Vol.3(3): 248-252 ISSN: 2010-3654DOI: 10.7763/IJEEEE.2013.V3.233.

[2] Aldiab, A., Chowdhury, H., Kootsookos, A., Alam, F., \& Allhibi, H. (2019). Utilization of Learning Management Systems (LMSs) in higher education system: A case review for Saudi Arabia. Energy Procedia, 160, 731-737. doi: 10.1016/j.egypro.2019.02.186.

[3] Alshorman, B. A., \& Bawaneh, A. K. (2018). Attitudes of Faculty Members and Students towards the Use of the Learning Management System in Teaching and Learning. Turkish Online Journal of Educational Technology-TOJET, 17(3), 1-15.

[4] Conradie, P. (2013). Applying system theory to develop a mobile learning pedagogical framework. Paper presented at 
the 82-XII. Retrieved from https://search.proquest.com/ docview/1380700191? accountid=33478.

[5] Garrison, G. D., PharmD., Baia, P., PhD., Canning, J. E., PharmD., \& Strang, A. F., PharmD. (2015). An asynchronous learning approach for the instructional component of a dual-campus pharmacy resident teaching program. American Journal of Pharmaceutical Education,79(2), 1-7. Retrieved from https://search.proque st.com/docview/1673370674? accountid $=33478$.

[6] Heidegger, M. (1954). The question concerning technology. Vorträge und Aufsätze.

[7] Karoudis, K., \& Magoulas, G. D. (2016). Ubiquitous learning architecture to enable learning path design across the cumulative learning continuum. Informatics, 3(4), 19. doi:http://dx.doi.org/10.3390/informatics3040019.

[8] Knowles, M. (1984) Andragogy in action: Applying modern principles of adult learning. San Francisco: Jossey-Bass.

[9] Kushrestha, T., \& amp; Kant, A. R. (2013). Benefits of learning management system (LMS) in Indian Education. International Journal of Computer Science \& Engineering Technology.

[10] Patel, H. I., \& Patel, D. (2017). Blended learning in higher education using MOODLE open source learning management tool. International Journal of Advanced Research in Computer Science,8(5) Retrieved from https://search.proquest.com/docview/1912631243?accounti $\mathrm{d}=33478$

[11] Stoica, G. A., \& Støckert, R. (2018). Designing new learning scenarios for multi-campus large classes in mathematics. Paper presented at the, 1495-500. doi:http://dx.doi.org/ 10.12753/2066-026X-18-070 Retrieved from https://search. 